\title{
Human brain activity associated with painful mechanical stimulation to muscle and bone
}

\author{
Lynn Maeda $\cdot$ Mayu Ono $\cdot$ Tetsuo Koyama • \\ Yoshitetsu Oshiro · Masahiko Sumitani · \\ Takashi Mashimo $\cdot$ Masahiko Shibata
}

Received: 8 February 2010/ Accepted: 12 May 2011/Published online: 2 June 2011

(C) Japanese Society of Anesthesiologists 2011

\begin{abstract}
Purpose The purpose of this study was to elucidate the central processing of painful mechanical stimulation to muscle and bone by measuring blood oxygen leveldependent signal changes using functional magnetic resonance imaging (fMRI).

Methods Twelve healthy volunteers were enrolled. Mechanical pressure on muscle and bone were applied at the right lower leg by an algometer. Intensities were adjusted to cause weak and strong pain sensation at either
\end{abstract}

L. Maeda

Department of Anesthesia and Pain Medicine, Nishinomiya

Municipal Central Hospital, Nishinomiya, Japan

L. Maeda · T. Mashimo

Department of Anesthesiology and Intensive Care Medicine,

Osaka University Graduate School of Medicine, Suita, Japan

M. Ono

Department of Anesthesia, Ikeda City Hospital,

Ikeda, Japan

T. Koyama

Department of Rehabilitation,

Kyoritsu Neurosurgery Hospital, Nishinomiya, Japan

Y. Oshiro

Himeji Ishikawa Functional Brain Imaging Laboratory,

Himeji, Japan

M. Sumitani

Department of Anesthesiology and Pain Relief Center,

The University of Tokyo Hospital, Tokyo, Japan

M. Shibata $(\bowtie)$

Department of Pain Medicine,

Osaka University Graduate School of Medicine,

2-2 Yamadaoka, Suita, Osaka 565-0871, Japan

e-mail:mshibata@pain.med.osaka-u.ac.jp target site in preliminary testing. Brain activation in response to mechanical nociceptive stimulation targeting muscle and bone were measured by fMRI and analyzed.

Results Painful mechanical stimulation targeting muscle and bone activated the common areas including bilateral insula, anterior cingulate cortex, posterior cingulate cortex, secondary somatosensory cortex (S2), inferior parietal lobe, and basal ganglia. The contralateral S2 was more activated by strong stimulation than by weak stimulation. Some areas in the basal ganglia (bilateral putamen and caudate nucleus) were more activated by muscle stimulation than by bone stimulation.

Conclusions The putamen and caudate nucleus may have a more significant role in brain processing of muscle pain compared with bone pain.

Keywords Pain $\cdot$ Muscle $\cdot$ Bone $\cdot$ Neuroimaging $\cdot$ fMRI

\section{Introduction}

Physical pain originating from deep tissues-including sprains, fibromyalgia, rheumatic polymyalgia, and other muscle-derived pain, and bone-derived pain such as fractures, spondylosis, and bone tumors-is very commonly encountered. Consequently, understanding how these conditions come to be painful through brain processing is clinically important. Recent imaging research using the blood oxygen level-dependent-based (BOLD) functional magnetic resonance imaging (fMRI) method successfully revealed cognitive mechanisms in response to painful stimulation.

In the field of anesthesiology, fMRI has been on trial as a tool for investigating cerebral pain processing [1-5]. Previous reports, however, have mainly been studies of 
heat stimulation to skin $[6,7]$. More recently, studies targeting brain activation when muscle pain is caused using electric stimulation or hypertonic saline have successfully demonstrated that some areas are differently activated, including the contralateral primary somatosensory cortex (S1), the ipsilateral anterior insula, the contralateral motor cortex, the cingulate motor area, and the perigenual cingulate [8-10]. However, there are few studies of the brain processing activated by mechanical stimulation to deep tissues [11]. To elucidate the central processing of painful mechanical stimulation to deep tissues (muscle and bone), we compared the brain activation induced by two different intensities of stimulation (strong and weak) at two different targets (muscle and bone), using fMRI.

\section{Materials and methods}

\section{Subjects}

All the procedures were approved by the Osaka University Hospital Institutional Review Board. Twelve healthy volunteers (7 men, 5 women; aged 24-56 years) agreed to receive painful stimulation while their brain activation was evaluated. They had no neurological disorders or detectable MRI abnormalities in the brain and were free from any medication within $24 \mathrm{~h}$ before the study. In written informed consent, each acknowledged that they were willing to receive experimental painful stimulation. Before the protocols were carried out, each volunteer was familiarized with the experimental protocol, the types of stimulation, and the tasks performed.

\section{Painful stimulation}

To determine suitable stimulation intensities for each subject, a preliminary testing was performed immediately

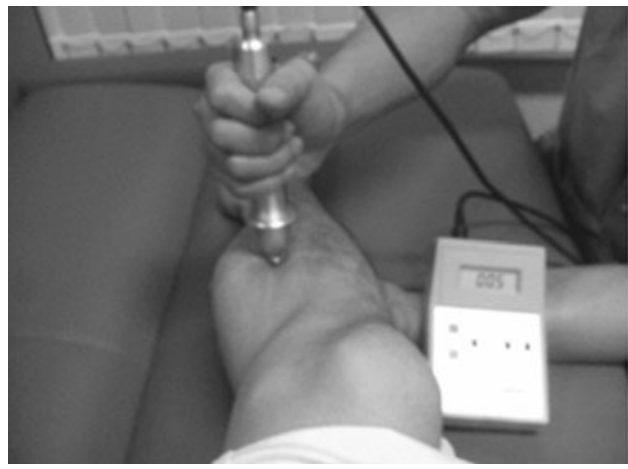

Fig. 1 Photograph showing how a digital algometer was used to apply stimulation to muscle (left) and bone (right). Bone stimulation was applied to the surface (skin) at the midpoint of tibia. Muscle stimulation was applied to the surface of gastrocnemius $3-5 \mathrm{~cm}$ before the fMRI study. Perpendicularly applying a round 10-mm solid tip of an algometer probe (Pressure Algometer NPA-1, Shinko, Japan) on the surface (skin) at the medial point of the right tibia (Fig. 1), the experimenter gradually increased the pressure until the subject verbally indicated that the stimulation was painful. At that point, the pain intensity was taken to be ' 3 ' on a subjective 10-point numerical rating scale (NRS). The three median values of five trials were averaged to determine the weak stimulation to be applied to the tibia of particular volunteers. Similarly, the subject was asked to verbally indicate when the pain was such that it would probably be intolerable for more than $20 \mathrm{~s}$ without withdrawal movement. At this point, pain intensity was scored as ' 8 ' on the volunteer's subjective 10-point NRS. Similar grading of muscle pain was also carried out [12]. Here the tip of the probe was applied to the skin on the gastrocnemius muscle at a medial point $3-5 \mathrm{~cm}$ from the stimulation point of the tibia (Fig. 1). For each volunteer, as in the bone protocol, subjective NRS pain scores of ' 3 ' and ' 8 ' were obtained. These procedures were conducted by one experimenter (M.S.) who, in each instance and so far as possible, endeavored to consistently apply the required level of pressure perpendicularly.

\section{Protocol}

Each subject participated in a trial comprising $12 \mathrm{fMRI}$ task sessions. At each right-leg site that was evaluated in the preliminary testing, three trials of $20 \mathrm{~s}$ of strong or weak stimulation were applied in each session. The site and strength of stimulation were pseudo random for each series. After each series of three stimulations (in a session lasting 3 min; Fig. 2), there was a pause of 1 min before the next session. MRI scans were acquired throughout the 36-min period. The subjects were asked to rate, using NRS, the overall pain at the end of each series of three stimulations (mean of three periods of 20-s stimulation). Before the

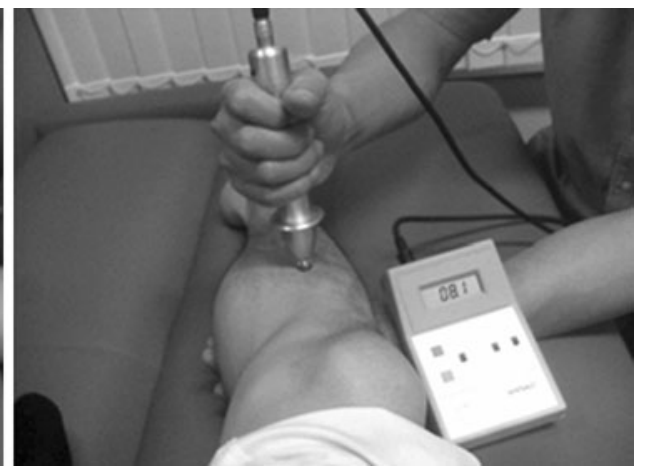

posterior from bone stimulation point. Intensities of stimulation (muscle vs. bone, strong vs. weak) were decided for each subject by averaging the values of the median of three of five trials 


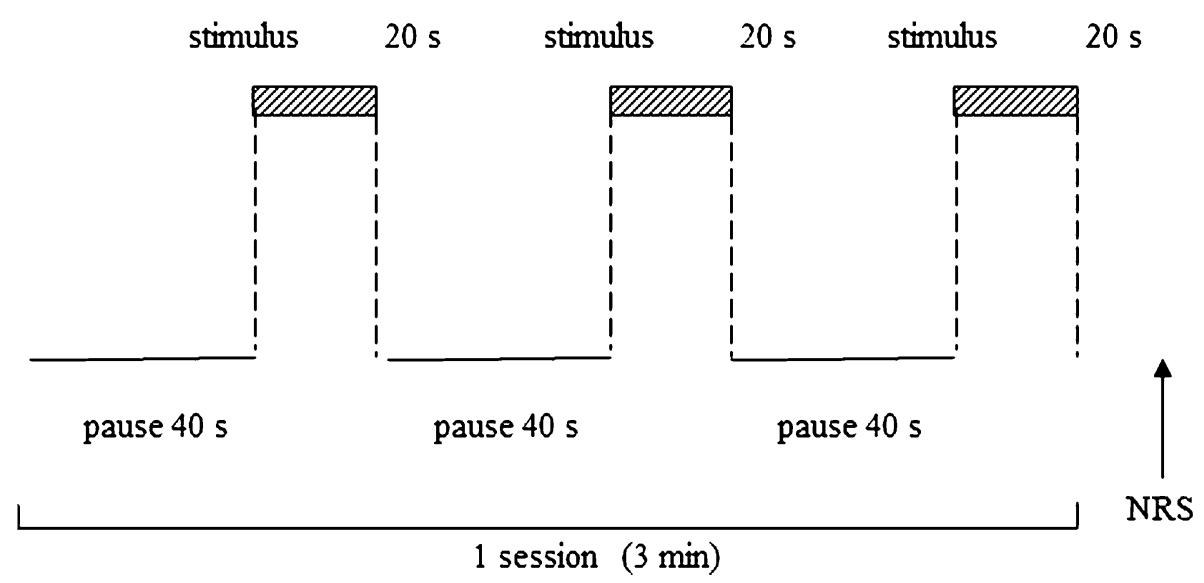

Fig. 2 Schematic representation of the experiment. Each subject underwent one trial comprising 12 sessions with a 1-min interval between sessions. Each session, lasting a total of $3 \mathrm{~min}$, started with 40 -s pause, and then a 20-s strong or weak stimulation to either

commencement of the protocol, each volunteer was informed that the site (muscle or bone) and intensity (weak or strong) of the forthcoming series of stimulation would be random.

\section{MRI}

Imaging was performed with a 1.5-T MRI scanner (Signa EXCITE XI 11.0; GE Healthcare, Milwaukee, WI, USA). Functional MR images were obtained using a multislice echo planar imaging technique (EPI) based on 30 oblique slices (repetition time, 3,000 ms; time to echo, $60 \mathrm{~ms}$; flip angle, $90^{\circ}$; slice thickness, $5 \mathrm{~mm}$; gap, $0 \mathrm{~mm}$; field of view, $300 \mathrm{~mm} \times 300 \mathrm{~mm}$; in-plane resolution, $4.69 \mathrm{~mm} \times$ $4.69 \mathrm{~mm}$ ). All the subjects were positioned in the scanner with a foam rubber pad to minimize head movement and instructed simply to lie with their eyes closed without moving or speaking. Their heads were placed so that the uppermost superior aspect of brain was within the field of view. High-resolution $\mathrm{T}_{1}$-weighted anatomic images with the same orientation as the EPI slices were collected from each subject. In the subsequent analysis, these images were used for coregistration of functional and anatomic data.

Data analysis

\section{Psychophysics}

Using paired $t$ testing, we compared the stimulation intensity and the average subjective NRS scores for muscle (weak and strong) and bone (weak and strong), respectively. Effects of order of stimulus application on NRS were evaluated with two-way analysis of variance (ANOVA); $P<0.05$ was regarded as significant. Values are given as mean $\pm \mathrm{SD}$. muscle or bone. Weak and strong stimulations for each volunteer were decided in the preliminary testing. The order of stimulation was pseudo randomized. After each session, volunteers were asked to score the perceived pain. $N R S$ numerical rating scale

fMRI data analysis

The fMRI data were analyzed with Statistical Parametric Mapping software (SPM99; Wellcome Department of Cognitive Neurology, London, UK) implemented in Matlab 6.1 (Mathworks, Sherborn, MA, USA). The functional images were realigned to correct for head movements, coregistered with each subject's anatomic MRI, and transformed to the format of the standard brain according to Talairach coordinates [13]. The functional images were spatially smoothed with an 8-mm full-width half-maximum Gaussian kernel. We fitted a linear regression model (fixed effects within subjects). Each condition was modeled with a boxcar function and convolved with a hemodynamic response function. Temporally, the voxel time series were high-pass filtered (124-s cutoff periods) to remove slow trends in the data, and low-pass filtered with a hemodynamic response filter.

We compared image data for each group-weak muscle stimulation, strong muscle stimulation; weak bone stimulation, strong bone stimulation - against the data from each resting condition, respectively. All the data were pooled for group statistical comparisons. Across the subjects, random effect analysis was performed to determine the significant activation associated with different sites and intensities of stimulation $(P<0.001$ uncorrected; minimum cluster size, 20 voxels). To investigate the brain network related to painful stimulation intensity, we calculated the difference in the areas activated by strong stimulation minus weak stimulation (paired $t$ test; $P<0.005$ uncorrected; minimum cluster size, 20 voxels). To identify the brain areas that are differently activated by muscle and bone stimulation, we also analyzed the data separately (strong muscle minus strong bone; weak muscle minus weak bone; and all 
together) (paired $t$ test; $P<0.005$ uncorrected; minimum cluster size, 20 voxels).

\section{Results}

Stimulation and pain intensity

The mean intensity of muscle stimulation was $21.1 \pm 8.4 \mathrm{~N}$ for weak stimulation and $40.7 \pm 7.8 \mathrm{~N}$ for strong stimulation; the mean intensity of bone stimulation was $14.2 \pm 5.7 \mathrm{~N}$ for weak stimulation and $30.1 \pm 7.9 \mathrm{~N}$ for strong stimulation. The NRS scores with strong muscle and strong bone stimulation were significantly higher than those with weak muscle and weak bone stimulation, respectively ( $P<0.05$ for both) (Table 1$)$. All the volunteers clearly distinguished the two intensities of stimulation because the weaker stimulation was always scored lower. Although the volunteers found weak muscle stimulation trials more painful $(P<0.05)$ than weak bone stimulation, no similar difference was found for strong stimulations. Comparing the data among the three trials of the same intensity at the same site, no differences in NRS scores were found; this indicates that repeated mechanical stimulation did not sensitize or desensitize the volunteers $(P>0.05)$.

\section{fMRI}

In response to painful muscle stimulation, brain activation was apparent within the bilateral anterior cingulate cortex (ACC), insula cortex, the secondary somatosensory cortex (S2), the inferior parietal lobule (IPL), the posterior cingulate cortex (PCC), putamen, the ipsilateral dorsolateral prefrontal cortex (DLPFC), thalamus, caudate, and the contralateral claustrum (Fig. 3a,b; Table 2). In response to painful bone stimulation, brain activation was also apparent within the bilateral ACC, the IPL, the S2, the PCC, the ipsilateral DLPFC, and the contralateral claustrum (Fig. 4a,b; Table 2). Peak coordinates $(x, y, z)$ in Montreal
Neurological Institute (MNI) space and Z-scores $(>6.00)$ for the activated brain regions by muscle and bone pain are shown in Table 2. Differences in the areas activated by strong versus weak muscle stimulation were mainly found in the contralateral S2 and the bilateral thalamus (Fig. 3c). For strong versus weak bone stimulation, the difference was found in the contralateral S2 (Fig. 4c). With weak stimulations, the activation differences between muscle and bone stimulation were apparent in the bilateral caudate nucleus and contralateral Brodmann areas 22 and 45 (Fig. 5a). With strong stimulations, the activation differences were apparent in the bilateral putamen, the ipsilateral ACC, and the contralateral claustrum (Fig. 5b). Analyzing the sum of the differences between muscle- and bonerelated pain revealed that activation was different in the bilateral caudate and contralateral putamen (Fig. 5c; Table 3).

\section{Discussion}

In this study, as an initial step toward elucidating the brain activation associated with mechanically induced deep tissue pain, we found that the contralateral S2 was more activated by stronger stimulations to muscle or bone. We also found that parts of the basal ganglia (putamen and caudate nucleus) were more activated by muscle stimulation than by bone stimulation.

\section{Psychophysics}

The NRS scores measured during fMRI were higher than those determined in the preliminary testing. Although the reason is not clear, the difference of the rate of pressure increase between preliminary testing and the fMRI session may be a cause.

On the other hand, the NRS scores for the two sets of three stimulations at each site did not change during the protocol (see Table 1), suggesting that no further change in sensitivity occurred. Nie et al. [14] have reported that after

Table 1 Numerical rating scale (0-10 NRS) by mechanical stimulation

\begin{tabular}{|l|l|l|l|l|}
\hline & 1 st stimulation & 2nd stimulation & 3rd stimulation & mean \\
\hline weak muscle stimulation & $4.83 \pm 1.99$ & $4.67 \pm 1.88$ & $4.5 \pm 1.98$ & $4.67 \pm 1.90$ \\
\hline strong muscle stimulation & $8.17 \pm 1.34$ & $8.5 \pm 1.17$ & $8.25 \pm 1.42$ & $8.31 \pm 1.28$ \\
\hline weak bone stimulation & $4.25 \pm 2.14$ & $3.25 \pm 1.14$ & $3.92 \pm 1.78$ & $3.81 \pm 1.74$ \\
\hline strong bone stimulation & $8.83 \pm 1.03$ & $8.25 \pm 1.36$ & $8.17 \pm 1.70$ & $8.42 \pm 1.38$ \\
\hline
\end{tabular}

NRS with strong muscle and strong bone stimulation were significantly higher than those with weak muscle and weak bone stimulation $(* P<0.05)$. In weak stimulations, NRS with muscle stimulation was higher than with bone stimulation $(* * P<0.05)$. There were no differences between strong muscle and bone stimulations among NRS with 1st, 2nd, and 3rd stimulation in the same study groups 
Fig. 3 Brain activation induced by muscle stimulation: weak stimulation (a), strong stimulation (b), and contrast of strong and weak stimulation (c). In millimeter elevations relative to a line through the anteriorposterior commissure (AC-PC line), brain slices are shown in superior-inferior sequence. The axial slices are arranged from dorsal (left) to ventral (right). Statistical map thresholds are $P<0.001$ (a, b, uncorrected), and $P<0.005$ (c, uncorrected, paired test). Minimum cluster size is 20 voxels. Right $(R)$ and left $(L)$ sides are indicated. $A C C$ anterior cingulate cortex; IPL inferior parietal lobule; $S 2$ secondary sensory cortex; $D L P F C$ dorsolateral prefrontal cortex; $P C C$ posterior cingulate cortex

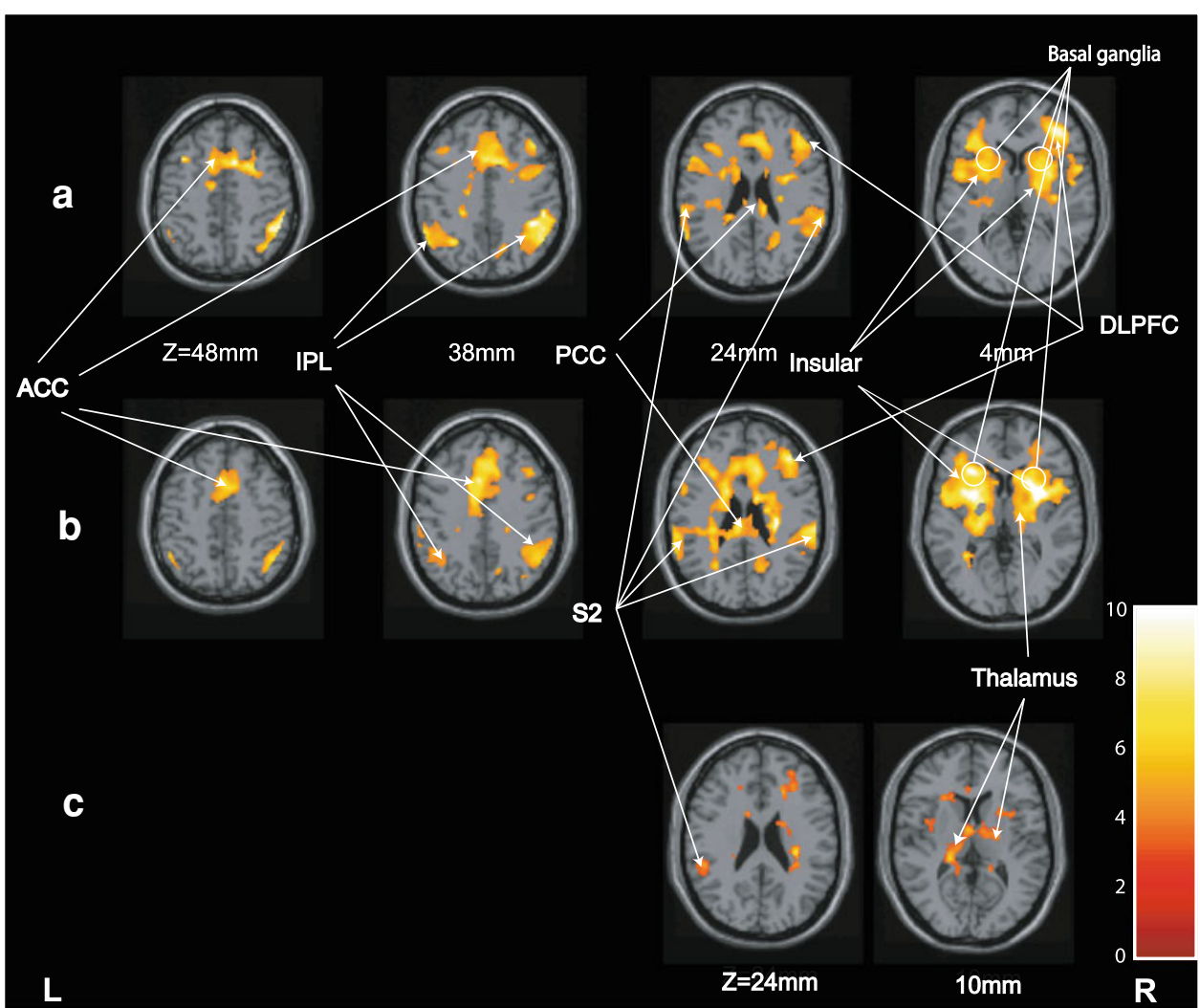

ten stimulations with 30-s intervals between stimulations, subjects gave higher VAS scores, increasing to $192 \% \pm 71 \%$ for stimulation of the tibia and to $117 \% \pm 42 \%$ for stimulation of the tibia anterior muscle. In the present study, the protocol specified a 60-s interval during all the sessions. This interval was apparently long enough to prevent temporal sensitization. To evoke equivalent pain in muscle and bone, greater stimulation had to be applied largely to the muscle; this may be because nociceptive nerve density is greater in the periosteum than in muscle [15].

\section{Imaging}

A previous positron emission tomography (PET) study and event-related fMRI study using noxious electronic stimulation of muscle showed activation in the ACC, S2, and anterior insula [8, 10]. Also, PET and fMRI study using injection of hypertonic saline into muscle evoked the activation in contralateral insula and putamen $[9,16]$. In our study, the results for mechanical stimulation are consistent with the previous muscle pain studies, which have shown that the different types of stimulation (hypertonic saline, electrical stimulation, and mechanical stimulation) applied to muscle induce equivalent activation patterns [8, $10,16]$. We found that stronger muscle stimulation resulted in greater activation in the contralateral S2, the bilateral thalamus (contralateral $>$ ipsilateral), and that stronger bone stimulation caused greater activation in the contralateral S2. In each case, the contralateral S2 was more activated by strong stimulation. However, caution is needed in interpreting these results because greater application of the force to skin is also involved during strong stimulation. The activation of the contralateral S2 may also come from greater cutaneous stimulation. In a PET study investigating thermal stimulation to skin, Coghill et al. [17] have shown that the ipsilateral cerebellum, the contralateral $\mathrm{S} 1$, the supplementary motor area, the bilateral S2, the lentiform nucleus, the insular cortex, and the thalamus and ACC are more activated depending on perceived pain intensity. In an fMRI study, using mechanical phasic stimulation on the skin, Ringler et al. [18] have shown that the contralateral $\mathrm{S} 2$ is more activated by stronger stimulation. The contralateral S2 may take part in processing pain intensity derived from both skin and deep tissues.

Comparison of the activation associated with muscle and bone stimulation revealed that caudate nucleus and putamen were more activated by muscle than by bone stimulation. No activation existed that was evoked by (bone $>$ muscle) stimulation (data not shown). The activation in these areas is supposed to be related to stimulated sites (muscle or bone), not to stimulation or pain intensity, because these areas were not included as a contrast when we stimulated the single target (muscle or bone) with the 
Table 2 Peak coordinates $(x, y$, $z$ ) in Montreal Neurological Institute (MNI) space and $Z$-scores (>6.00) for the activated brain regions by muscle and bone pain

Brodmann areas are given where available

$B A$ Broadmann area, $A C C$ anterior cingulate cortex, $S 2$ secondary sensory cortex, IPL inferior parietal lobule

Fig. 4 Brain activation induced by bone stimulation: weak stimulation (a), strong stimulation (b), and contrast of strong and weak stimulation (c). In millimeter elevations relative to a line through the anteriorposterior commissure (AC-PC line), brain slices are shown in superior-inferior sequence. The axial slices are arranged from dorsal (left) to ventral (right). Statistical map thresholds are $P<0.001$ (a, b, uncorrected), and $P<0.005$ (c, uncorrected, paired test). Minimum cluster size is 20 voxels. Right $(R)$ and left $(L)$ sides are indicated. $A C C$ anterior cingulate cortex; IPL inferior parietal lobule; $S 2$ secondary sensory cortex; $D L P F C$ dorsolateral prefrontal cortex; $P C C$ posterior cingulate cortex

\begin{tabular}{|c|c|c|c|c|c|c|c|c|c|c|}
\hline \multirow[t]{3}{*}{ Region } & \multirow[t]{3}{*}{ BA } & \multicolumn{4}{|c|}{ Muscle pain } & \multirow[t]{3}{*}{ BA } & \multicolumn{4}{|c|}{ Bone pain } \\
\hline & & \multicolumn{3}{|c|}{ Peak voxel coordinate } & \multirow[t]{2}{*}{$Z$-score } & & \multicolumn{3}{|c|}{ Peak voxel coordinate } & \multirow[t]{2}{*}{$Z$-score } \\
\hline & & $x$ & $y$ & $z$ & & & $x$ & $y$ & $z$ & \\
\hline \multirow[t]{3}{*}{ ACC } & 32 & -8 & 12 & 44 & 7.07 & & & & & \\
\hline & 32 & -6 & 18 & 32 & 6.42 & & & & & \\
\hline & 32 & -12 & 26 & 30 & 6.42 & & & & & \\
\hline \multirow[t]{2}{*}{ Insula right } & 13 & 28 & 26 & 2 & 6.33 & & & & & \\
\hline & & 34 & 4 & 8 & 6.09 & & & & & \\
\hline \multirow[t]{2}{*}{ Insula left } & & -34 & 6 & 8 & 6.25 & 44 & -48 & 10 & 6 & 6.27 \\
\hline & 47 & -28 & 24 & -8 & 6.05 & & & & & \\
\hline S2 right & 40 & 62 & -32 & 30 & 6.32 & & & & & \\
\hline S2 left & 40 & -60 & -32 & 28 & 6.39 & & & & & \\
\hline \multirow[t]{3}{*}{ IPL left } & & & & & & 40 & -48 & -52 & 40 & 6.57 \\
\hline & 40 & -48 & -58 & 42 & 6.33 & 40 & -48 & -40 & 28 & 6.48 \\
\hline & 40 & -44 & -48 & 42 & 6.13 & 40 & -62 & -36 & 32 & 6.27 \\
\hline Thalamus right & & 20 & -18 & 10 & 6.04 & & & & & \\
\hline \multirow[t]{2}{*}{ Putamen right } & & 26 & 2 & 6 & 6.16 & & & & & \\
\hline & & 20 & -4 & 8 & 6.10 & & & & & \\
\hline Putamen left & & -32 & 6 & 6 & 6.54 & & & & & \\
\hline Claustrum left & & -26 & 12 & 10 & 6.39 & & -30 & 10 & 6 & 6.18 \\
\hline Caudate right & & 16 & 12 & 8 & 6.13 & & & & & \\
\hline
\end{tabular}

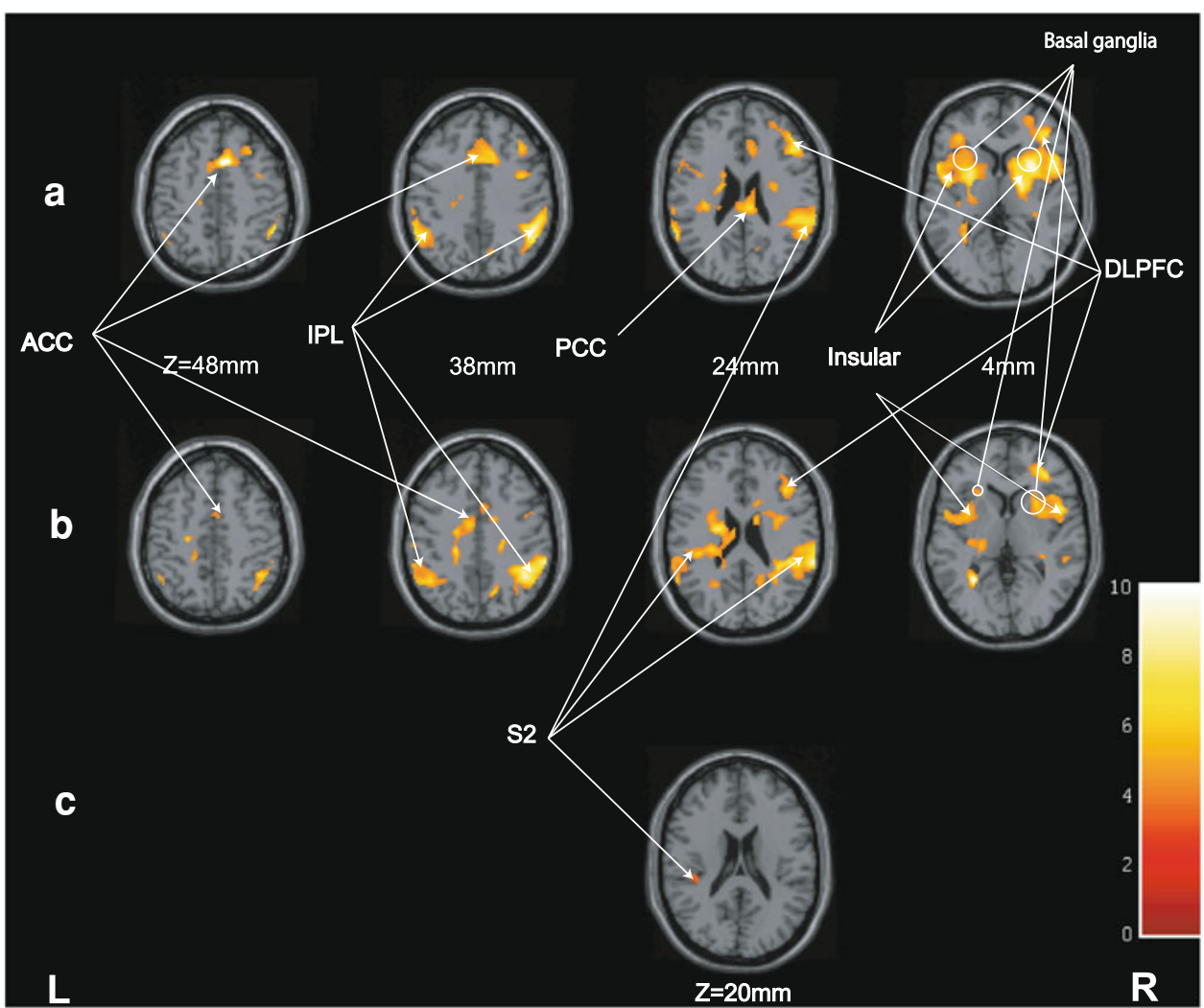

different intensities. Additionally, pain intensities were identical between strong muscle and strong bone pain. Reports of the previous pain imaging studies have suggested that the putamen is activated by nociceptive stimulation [17, 19], including muscle pain with hypertonic saline [16]. Basal ganglia are reported to have a role in 
Fig. 5 Contrast of brain activation induced by muscle and bone stimulation: contrast of weak stimulation to muscle and bone (a), contrast of strong stimulation to muscle and bone (b), and contrast of all the stimulations to muscle and bone (c). In millimeter elevations relative to a line through the anterior-posterior commissure (AC-PC line), brain slices are shown in superior-inferior sequence. The axial slices are arranged from dorsal (left) to ventral (right). Statistical map thresholds are $P<0.005$ (uncorrected, paired test). Minimum cluster size is 20 voxels. Right $(R)$ and left $(L)$ sides are indicated. $B A$ Brodmann area; $A C C$ anterior cingulate cortex

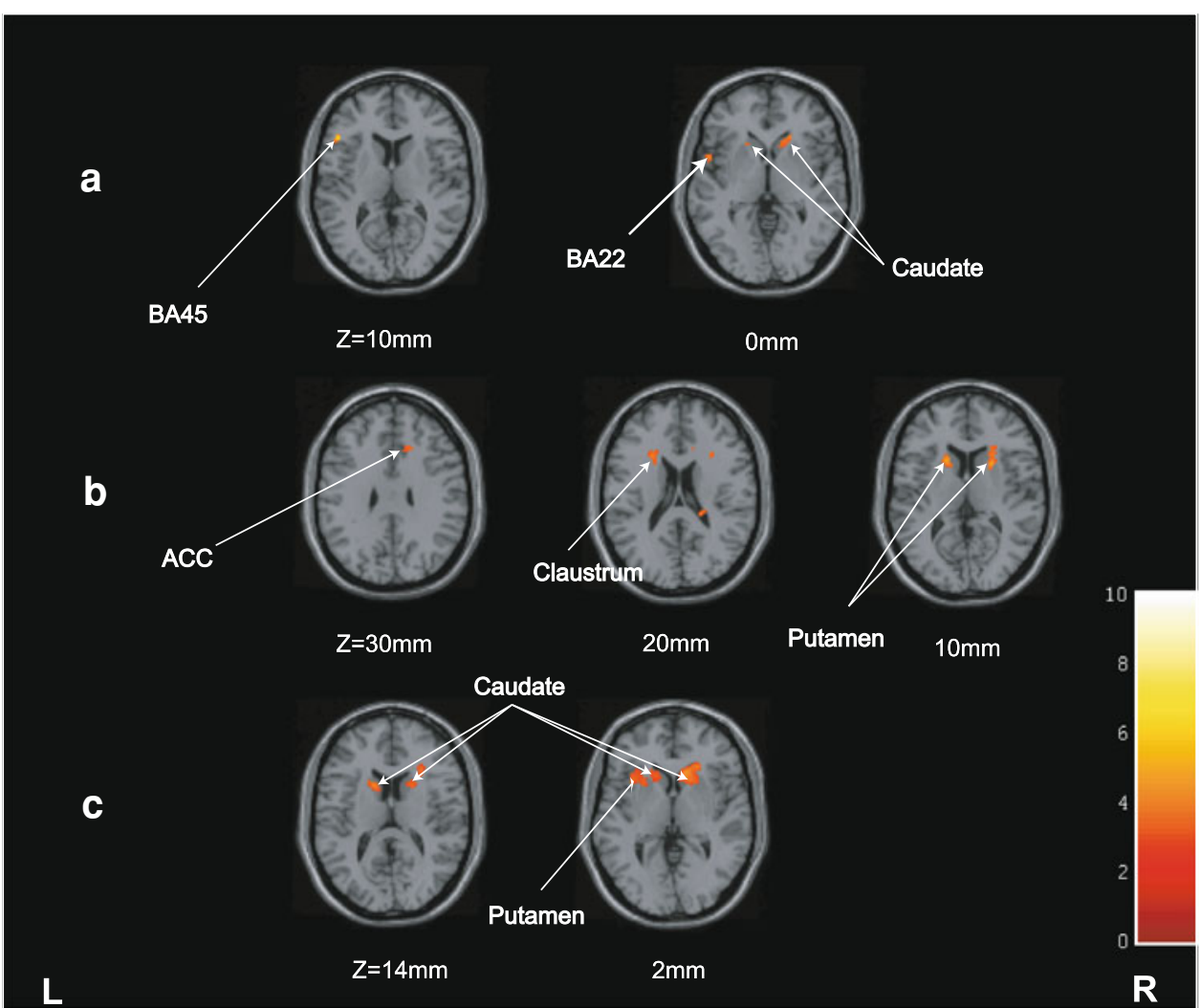

Table 3 Peak coordinates $(x, y, z)$ in Montreal Neurological Institute (MNI) space and $Z$-scores for activated brain regions by muscle-bone pain

\begin{tabular}{lllrl}
\hline Region & \multicolumn{2}{l}{ (Muscle-bone) pain } & \\
\cline { 2 - 4 } & \multicolumn{2}{l}{ Peak voxel coordinate } & \\
\cline { 2 - 4 } & $x$ & $y$ & $z$ & \\
\hline Putamen left & -24 & 12 & 24 & 4.12 \\
Caudate left & -18 & 16 & 8 & 4.01 \\
Caudate right & 16 & 22 & 0 & 3.95 \\
\hline
\end{tabular}

motor preparation, movement control, and emotional, motivational, and cognitive function [20-22]. Processing in the putamen and caudate nucleus is also reported to be related to pain-avoidance behavior [23]. Our results suggest that the putamen and caudate nucleus may have a more significant role in the brain processing of muscle pain compared with bone pain.

\section{Conclusion}

In conclusion, the putamen and caudate nucleus may have a more significant role in the brain processing of muscle pain compared to bone pain.
Acknowledgment We thank Syoji Kobashi and Tamotsu Nomura for helping in imaging procedures, Tateo Iwakura for help with image analysis, and Sho Karl Shibata and David Eunice for editing. Scans were performed at Ishikawa Hospital. This work was supported by a Grant-in-aid for community health and medical care from the Ichou Association for Promotion of Medical Science.

Conflict of interest None.

\section{References}

1. Hoffman HG, Richards TL, Van Oostrom T, Coda BA, Jensen MP, Blough DK, Sharar SR. The analgesic effects of opioids and immersive virtual reality distraction: evidence from subjective and functional brain imaging assessments. Anesth Analg. 2007; 105:1776-83.

2. Buvanendran A, Ali A, Stoub TR, Berger RA, Kroin JS. The use of brain positron emission tomography to identify sites of postoperative pain processing with and without epidural analgesia. Anesth Analg. 2007;105:1784-6.

3. Liu SS, Brown EN. Will seeing become believing? Anesth Analg. 2007;105:1526-7.

4. Lorenz IH, Egger K, Schubert H, Schnurer C, Tiefenthaler W, Hohlrieder M, Schocke MF, Kremser C, Esterhammer R, Ischebeck A, Moser PL, Kolbitsch C. Lornoxicam characteristically modulates cerebral pain-processing in human volunteers: a functional magnetic resonance imaging study. $\mathrm{Br} \mathrm{J}$ Anaesth. 2008; 100:827-33. 
5. Kurata J. A potential role of functional magnetic resonance imaging in the diagnosis of pain. Jpn J Anesthesiol. 2009;58: 1350-9. (in Japanese with English abstract).

6. Seifert F, Jungfer I, Schmelz M, Maihofner C. Representation of UV-B-induced thermal and mechanical hyperalgesia in the human brain: a functional MRI study. Hum Brain Mapp. 2008; 29:1327-42.

7. Borsook D, Moulton EA, Tully S, Schmahmann JD, Becerra L. Human cerebellar responses to brush and heat stimuli in healthy and neuropathic pain subjects. Cerebellum. 2007;22:1-21.

8. Niddam DM, Yeh TC, Wu YT, Lee PL, Ho LT, Arendt-Nielsen L, Chen AC, Hsieh JC. Event-related functional MRI study on central representation of acute muscle pain induced by electrical stimulation. Neuroimage. 2002;17:1437-50.

9. Henderson LA, Bandler R, Gandevia SC, Macefield VG. Distinct forebrain activity patterns during deep versus superficial pain. Pain. 2006;120:286-96.

10. Svensson P, Minoshima S, Beydoun A, Morrow TJ, Casey KL. Cerebral processing of acute skin and muscle pain in humans. J Neurophysiol. 1997;78:450-60.

11. Kobayashi Y, Kurata J, Sekiguchi M, Kokubun M, Akaishizawa T, Chiba Y, Konno S, Kikuchi S. Augmented cerebral activation by lumbar mechanical stimulus in chronic low back pain patients: an FMRI study. Spine. 2009;34:2431-6.

12. Gracely RH, Petzke F, Wolf JM, Clauw DJ. Functional magnetic resonance imaging evidence of augmented pain processing in fibromyalgia. Arthritis Rheum. 2002;46:1333-43.

13. Talairach J, Tournoux P. Co-planar stereotactic atlas of the human brain: 3-dimensional proportional system-an approach to cerebral imaging. New York: Thieme; 2002.

14. Nie H, Arendt Nielsen L, Andersen H, Graven Nielsen T. Temporal summation of pain evoked by mechanical stimulation in deep and superficial tissue. J Pain. 2005;6:348-55.
15. Mach DB, Rogers SD, Sabino MC, Luger NM, Schwei MJ, Pomonis JD, Keyser CP, Clohisy DR, Adams DJ, O'Leary P, Mantyh PW. Origins of skeletal pain: sensory and sympathetic innervation of the mouse femur. Neuroscience. 2002;113:155-66.

16. Korotkov A, Radovanovic S, Ljubisavljevic M, Lyskov E, Kataeva G, Roudas M, Pakhomov S, Thunberg J, Medvedev S, Johansson H. Comparison of brain activation after sustained nonfatiguing and fatiguing muscle contraction: a positron emission tomography study. Exp Brain Res. 2005;163:65-74.

17. Coghill RC, Sang CN, Maisog JM, Iadarola MJ. Pain intensity processing within the human brain: a bilateral, distributed mechanism. J Neurophysiol. 1999;82:1934-43.

18. Ringler R, Greiner M, Kohlloeffel L, Handwerker HO, Forster C. BOLD effects in different areas of the cerebral cortex during painful mechanical stimulation. Pain. 2003;105:445-53.

19. Bingel U, Glascher J, Weiller C, Buchel C. Somatotopic representation of nociceptive information in the putamen: an eventrelated fMRI study. Cereb Cortex. 2004;14:1340-5.

20. Doyon J, Bellec P, Amsel R, Penhune V, Monchi O, Carrier J, Lehericy S, Benali H. Contributions of the basal ganglia and functionally related brain structures to motor learning. Behav Brain Res. 2009;199:61-75.

21. Marchand WR. Cortico-basal ganglia circuitry: a review of key research and implications for functional connectivity studies of mood and anxiety disorders. Brain Struct Funct. 2010;215:73-96.

22. Grahn JA, Parkinson JA, Owen AM. The role of the basal ganglia in learning and memory: neuropsychological studies. Behav Brain Res. 2009;199:53-60.

23. Chudler EH, Dong WK. The role of the basal ganglia in nociception and pain. Pain. 1995;60:3-38. 